\title{
Endometrial Stromal Nodule: A Case Report
}

\author{
Şerife Özlem Genç, MD, Aliye Nigar Serin, MD* and Burcu Yılmaz, MD \\ ${ }^{1}$ Department of Obstetrics and Gynecology, Aksaray University, Turkey \\ ${ }^{2}$ Department of Gynecology and Obstetrics, Karamanoglu Mehmetbey University, Karaman, Turkey \\ ${ }^{3}$ Department of Pathology, Karamanoglu Mehmetbey University, Turkey
}

*Corresponding author: Aliye Nigar Serin, MD, Department of Gynecology and Obstetrics, Karamanoglu Mehmetbey University, Karaman, Turkey, Postal code: 70200, Tel: +90-530-580-3092

\begin{abstract}
The endometrial stromal nodule (ESN) is the rarest subtype of endometrial stromal tumors (ESTs) that's why experience with ESN is limited. ESN could mimic highly cellular leiomyomas, usually seen in the premenopausal age. Patients present with non-specific symptoms such as abnormal bleeding, lower abdominal pain, or maybe asymptomatic. Our patient was 46 years old women suffering from abnormal uterine bleeding and lower abdominal pain and had no desire to protect fertility. The preoperative diagnosis of ESN is very difficult. Also, no specific imaging is defined for the ESN in ultrasound or MRI examination. ESN is usually misdiagnosed as uterine fibroids, interstitial ectopic pregnancy, or stromal hyperplasia. Macroscopically, these tumors are classically well-circumscribed, solitary, encapsulated. In a curettage specimen, the distinction between an ESN and a low-grade endometrial stromal sarcoma is almost impossible, unless the tumor is too small, and the margins can be fully evaluated. In conclusion, ESN is a rare subtype of endometrial stromal sarcomas, but there is nothing to afraid of if you do the hysterectomy. So, it is important to diagnose preoperatively especially in young nulliparous women who desire to preserve fertility. Clinical presentation is non-specific and there is no preoperative imaging that can completely rule out malignancy. It is necessary to develop new diagnostic methods or new treatment options to protect the uterus.
\end{abstract}

\section{Keywords}

Endometrial stromal tumor, Endometrial stromal nodule, Leiomyoma, Highly cellular myoma, Uterine sarcoma

\section{Introduction}

Endometrial stromal tumors (ESTs) are very rare mesenchyme tumors, and the endometrial stromal nodule (ESN) is the least common [1]. 2014 WHO classifica- tion divides ESTs into four groups: Endometrial stromal nodule, low-grade endometrial stromal sarcoma, highgrade endometrial stromal sarcoma, and undifferentiated uterine sarcoma [2]. However, the ESN is difficult to diagnose because of its similarity to low grade endometrial stromal sarcomas [3]. The ESN is noninvasive and clinically benign type of ESTs.

There is limited information about the ESN because of its rarity. The definitive diagnosis of the ESN depends on an attentive examination of the tumor margin after hysterectomy.

\section{Case}

We report a 46-year-old woman as a case who had abnormal uterine bleeding and lower abdominal pain. Transvaginal ultrasound scan revealed a corpus luteum cyst in the right ovary and well-circumscribed fibromas; the bigger one of which was $6 \times 6 \mathrm{~cm}$. The intrauterine device had been applied to the patient for the treatment of abnormal bleeding but failed. After that, the endometrial curettage examination showed late proliferative endometrium. As she had no fertility desire, she underwent a hysterectomy and bilateral salpingo-oophorectomy because of menometrorrhagia. After histopathological examination, was defined as an endometrial stromal nodule.

On macroscopic examination, in the sections of the uterus, 5 nodules, the largest of which was $6 \mathrm{~cm}$ and the smallest of which was $0.5 \mathrm{~cm}$ in diameter, were detected. As in this case, the biopsy may be negative when the tumor is not associated with the endometrium. The

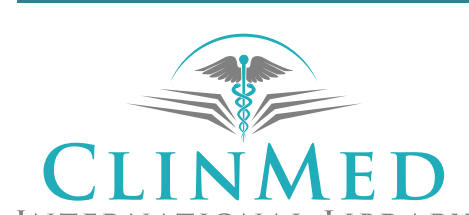

INTERNATIONAL LIBRARY
Citation: Genç SO, Serin AN, YıImaz B (2021) Endometrial Stromal Nodule: A Case Report. Obstet Gynecol Cases Rev 8:200. doi.org/10.23937/2377-9004/1410200

Accepted: April 22, 2021: Published: April 24, 2021

Copyright: (C) 2021 Genç SO, et al. This is an open-access article distributed under the terms of the Creative Commons Attribution License, which permits unrestricted use, distribution, and reproduction in any medium, provided the original author and source are credited. 


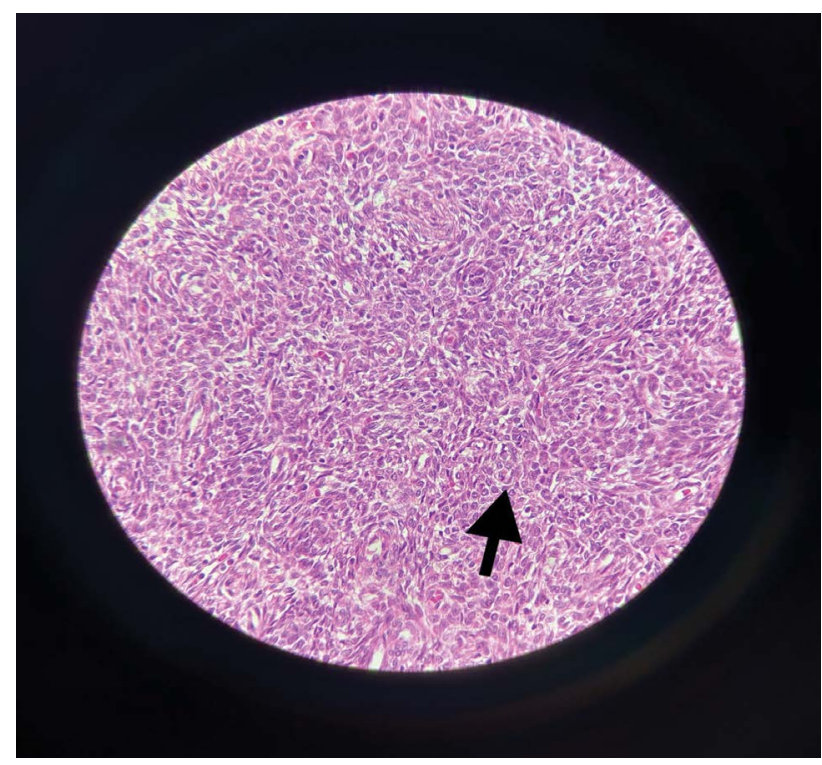

Figure 1: Diffuse growth of uniform small cells that resemble proliferative-phase endometrial stroma. ( $\times 400, \mathrm{H} \& \mathrm{E})$.

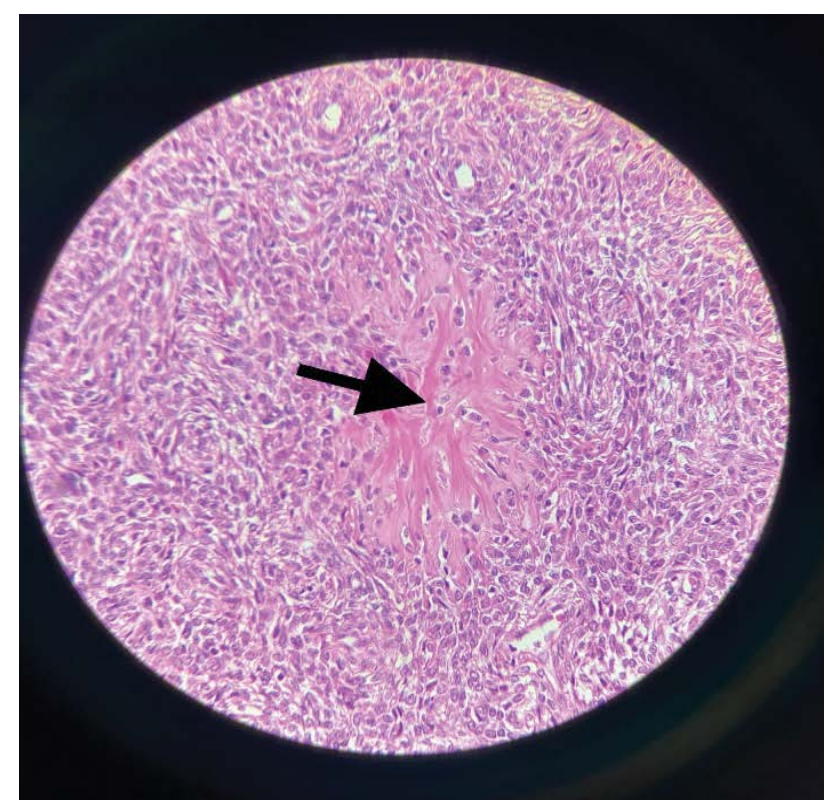

Figure 2: Smooth muscle differentiation displaying a starburst morphology (arrowed). (× 400, H\&E).

largest nodule was intramural with well-circumscribed and $6 \times 6 \times 5 \mathrm{~cm}$ in diameter. Its cut surface was fleshy, tan, focal orange colored and had a fibrillary configuration.

Histomorphological findings showed that nodule has a non-infiltrative border and consists of uniform small cells with oval-round, blue, basophilic nucleus with chromatin which is finely granular, has an indeterminate nucleolus, and a scant cytoplasm (Figure 1 and Figure 2). Tumor cells expressed diffuse CD10 and weakly SMA positivity (Figure 3 ).

The lesion was in cellular morphology and there was no cellular atypia, mitosis or necrosis. Hyalinized smooth muscle differentiation which expressed desmin and caldesmon (Figure 4 and Figure 5) and MTK positiv-

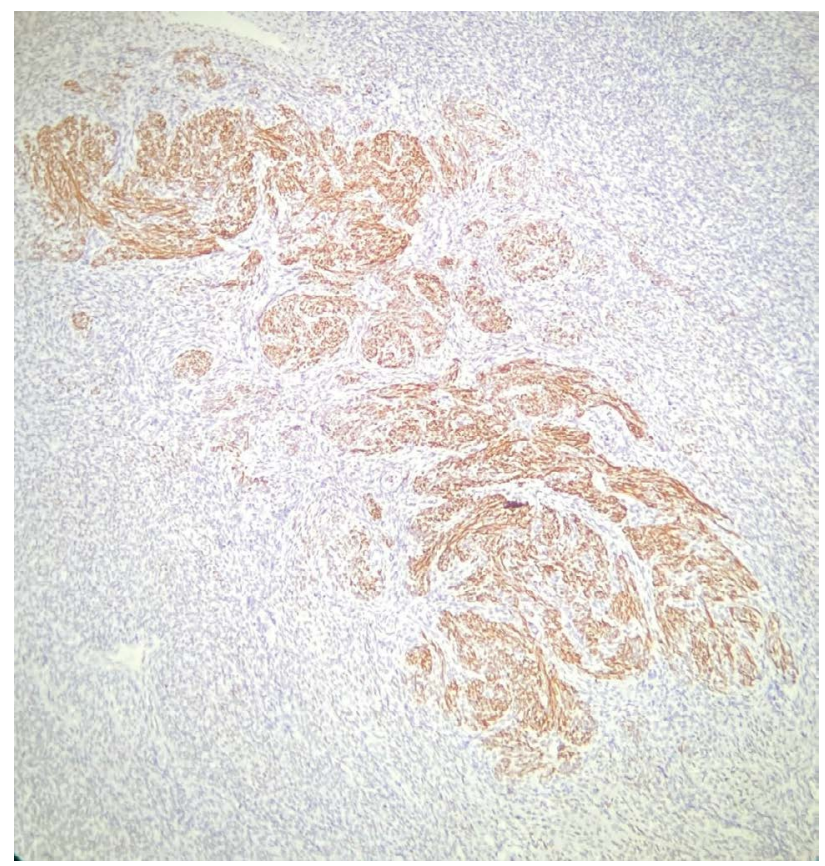

Figure 3: CD10 shows mostly positivity in uniform cells and negativity on the center which displays smooth muscle differentiation called starburst morphology ( $\times 200$, CD10).

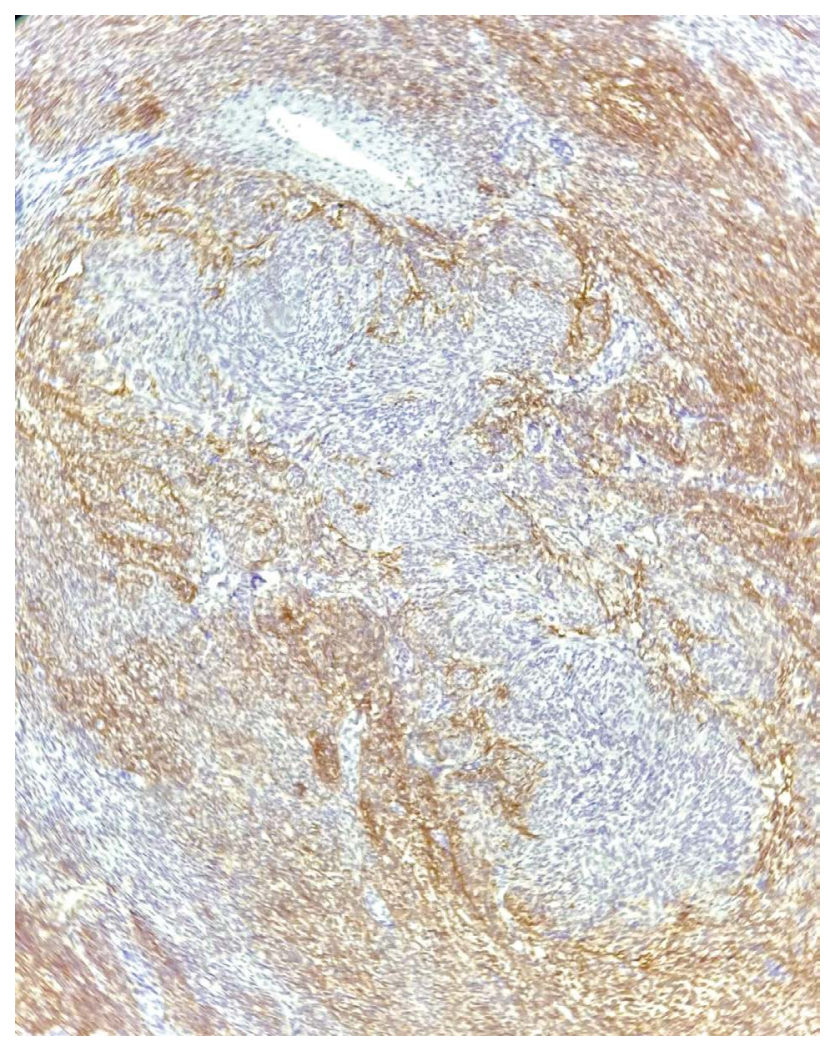

Figure 4: Focal smooth muscle differentiation shows typical Desmin expression, serially cut section of CD10 immuno staining ( $\times 200$, Desmin).

ity showing starburst morphology was observed in the focal areas (Figure 6). Starburst pattern is composed of smooth muscle differentiation with central hyalinisation and radiating collagen bants at the periphery encircle round cells (Figure 2). There was no lymphatic, vascular or myometrial invasion. 


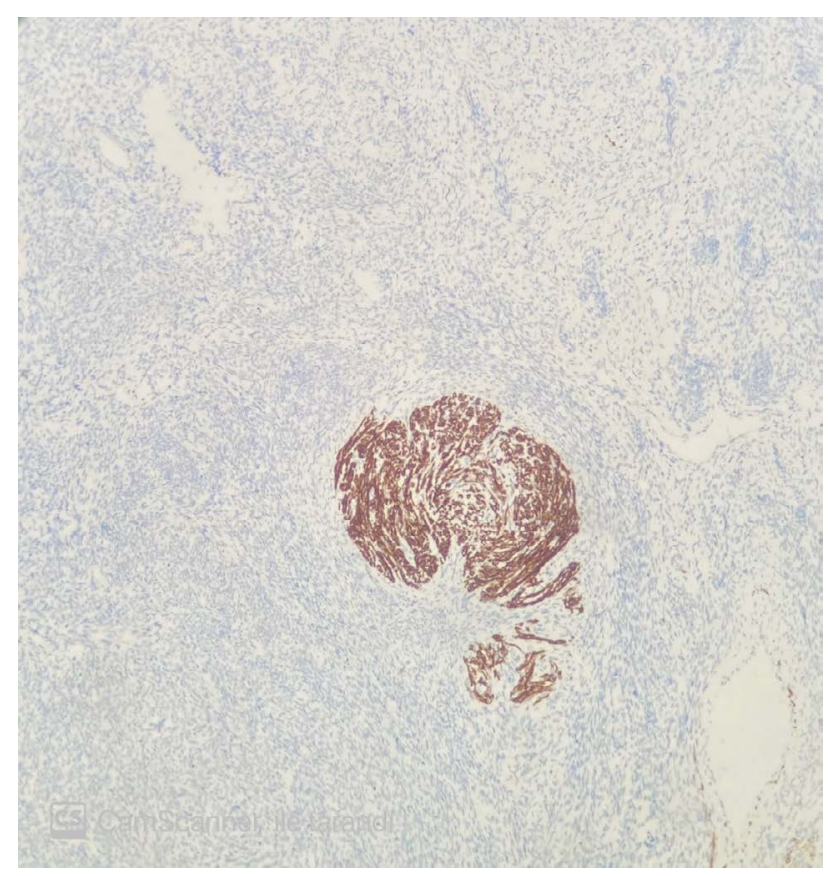

Figure 5: Caldesmon shows smooth muscle differentiation of starburst morphology. ( $\times 200$, Caldesmon).

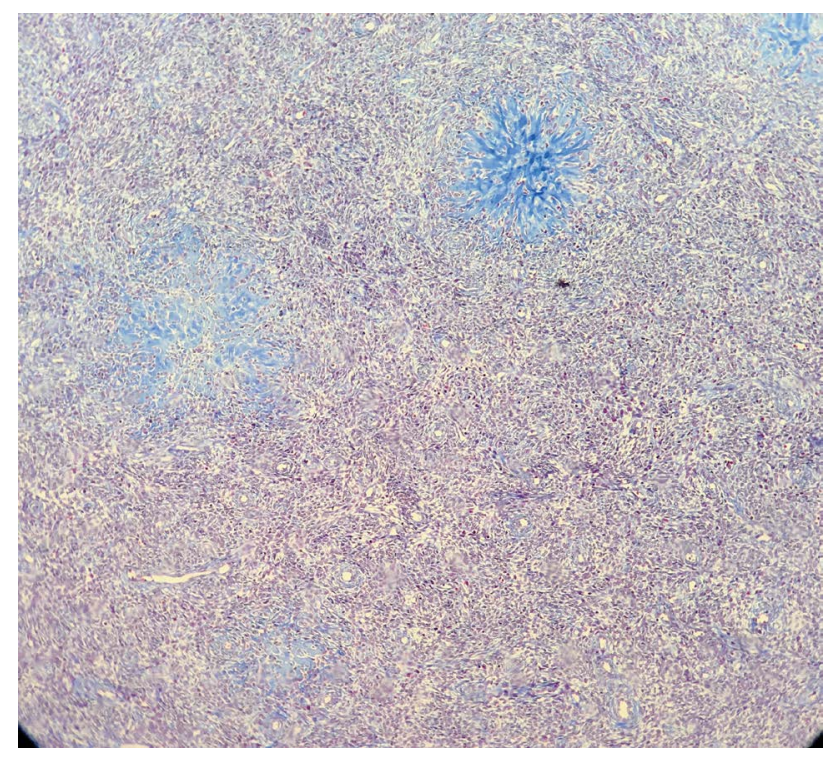

Figure 6: MTK revealed starburst morphology ( $\times 200$, MTK).

\section{Discussion}

Experience with endometrial stromal nodules (ESNs) is limited due to less than $2 \%$ of all uterine neoplasms and the literature on these lesions is inadequate. There is only one large series of ESNs which is reported with 60 cases in 1981 [4]. Recently, Dionigi published a series of 50 cases with endometrial stromal tumors which includes only 4ESNs [5].

ESN usually occurs at premenopausal age, but can be observed over a wide age range, such as 23 to 86 years [5]. Our case was a 46 year-old perimenopausal woman and there was no need to protect fertility.

The preoperative diagnosis of ESNs is very difficult. Patients may have abnormal bleeding, lower abdomen, or pelvic pain. However, they can sometimes be asymptomatic [5]. Our patient had lower pain and abnormal vaginal bleeding. Also, no specific medical imaging is defined for an ESN in ultrasound or MRI examination so postoperative pathology is essential for the diagnosis of an ESN. The ESN is usually misdiagnosed as uterine fibroids, interstitial ectopic pregnancy or stromal hyperplasia. These tumors are well-circumscribed, solitary, encapsulated and usually found in myometrium with a size of 1 to $22 \mathrm{~cm}$ and an average size of $7 \mathrm{~cm}$, but may also be located across endometrium as a polypoid mass $[3,4]$. In this case, the transvaginal ultrasound showed a few well-circumscribed masses, the biggest of which one was $6^{*} 6 \mathrm{~cm}$, which might be fibroids. ESN cases are generally diagnosed preoperatively as leiomyoma and adnexal mass [6]. Our preoperative diagnosis was leiomyoma in line with the literature, but postoperative pathology confirmed the ESN. In an endometrial biopsy material, the differences between an ESN and a low grade endometrial stromal sarcoma is almost impossible unless the tumor is too small, and the borders are fully evaluated. In our case endometrial biopsy was diagnosed as late proliferative endometrium.

Differential diagnosis of endometrial stromal nodules completely depends on the histopathological findings. Multiple preparate examination and serial cut sections must be performed. These stromal nodules have expansile, non-infiltrative margins with adjacent myometrium [7]. Lymphatic and vascular invasion is absent. Immunhistochemical expression includes CD10 positivity, SMA, caldesmon and desmine negativity (or weakly positivity).

In our case, the tumor expresses diffuse CD10. Only starburst pattern showed Desmin and Caldesmon positivity. The nodule was immunonegative with desmine and caldesmon in the regions other than these starburst areas.

Although ESNs are benign tumors, hysterectomy is the gold standard for total histological examination of the uterus to rule out malignancy [8]. On the other hand, Schilder, et al. published a case report involving a successful hormone therapy of the endometrial stromal nodule to maintain fertility in a young nulliparous woman [9]. As a result, local excision and hormonal therapy can be successful in women of reproductive age who want to maintain fertility. Hysteroscopy can then be used to evaluate the full myometrial interface and to monitor tumor growth [10].

In conclusion, it is not easy to diagnose the ESN preoperatively because of its similarity to cellular leiomyoma. If the postoperative result shows the ESN, the prognosis is excellent. The gold standard is to perform hysterectomy in patients diagnosed with the ESN. This rare tumor should be considered among the differential diagnoses in patients with irregular vaginal bleeding and uterine fibroma. Since there is a few ESN in the litera- 
ture, lots of studies are needed, which may improve the knowledge about the preoperative diagnosis or treatment and surgery options.

\section{Consent to Participate}

The written informed consent was obtained from the patient to publish case characteristics.

\section{Consent for Publication}

Not applicable.

\section{Availability of Data and Materials Statement}

The data sets used and/or analysed during the current study are available from the corresponding author on reasonable request.

\section{Competing Interests}

No potential conflict of interest was reported by the author(s). The authors alone are responsible for the content andwriting of the article.

\section{Funding}

None

\section{References}

1. Fdili Alaoui FZ, Chaara $H$, Bouguern $H$, Melhouf MA, Fatemi H, et al. (2011) Endometrial stromal nodule: Report of a case.

2. Ali RH, Rouzbahman M (2015) Endometrial stromal tumours revisited: An update based on the 2014 WHO classification. J Clin Pathol 68: 325-332.
3. Manuel V, Dinato FJ, Gutierrez PS, Siqueira ACS, Gaiotto FA, et al. (2017) Cardiac metastatic endometrial stromal sarcoma 17 years after hysterectomy. J Card Surg 32: 636638.

4. Tavassoli FA, Norris HJ (1981) Mesenchymal tumours of the uterus. VII. A clinicopathological study of 60 endometrial stromal nodules. Histopathology 5: 1-10.

5. Dionigi A, Oliva E, Clement PB, Young R (2002) Endometrial stromal nodules and endometrial stromal tumors with limited infiltration: A clinicopathologic study of 50 cases. Am J Surg Pathol 26: 567-581.

6. Elagoz S, Kıvanc F, Aker H (2005) Endometrial stromal tumors - A report of 5 cases. Aegean Pathology Journal 140-145.

7. J An, G Ruan, XY Xie, Shi YQ, Mao XD, et al. (2020) Endometrial stromal nodule: Report of 8 cases and literature review. European Journal of Gynaecological Oncology 41: 332-338.

8. Ferreira J, Felix A, Lennerz JK, Oliva E (2018) Recent advances in the histological and molecular classification of endometrial stromal neoplasms. Virchows Arch 473: 665678.

9. Schilder JM, Hurd WW, Roth LM, Sutton GP (1999) Hormonal treatment of an endometrial stromal nodule followed by local excision. Obstet Gynecol 93: 805-807.

10. Vilos GA, Harding PG, Sugimoto AK, Ettler HAC, Bernier MJ (2001) Hysteroscopic endomyometrial resection of three uterine sarcomas. J Am Assoc Gynecol Laparosc 8: 545-551. 